\title{
Clinicopathological Characteristics and Disease-Free Survival in Patients with Hürthle Cell Carcinoma: A Multicenter Cohort Study in South Korea
}

\author{
Meihua Jin ${ }^{1, *}$, Eun Sook Kim²,*, Bo Hyun Kim³ ${ }^{3}$, Hee Kyung Kim ${ }^{4}$ Yea Eun Kang ${ }^{5}$, Min Ji Jeon ${ }^{1}$, Tae Yong Kim ${ }^{1}$, \\ Ho-Cheol Kang ${ }^{4}$, Won Bae Kim ${ }^{1}$, Young Kee Shong ${ }^{1}$, Mijin Kim³ ${ }^{3}$, Won Gu Kim ${ }^{1}$ \\ ${ }^{1}$ Division of Endocrinology and Metabolism, Department of Internal Medicine, Asan Medical Center, University of Ulsan \\ College of Medicine, Seoul; ${ }^{2}$ Department of Internal Medicine, Ulsan University Hospital, University of Ulsan College of \\ Medicine, Ulsan; ${ }^{3}$ Division of Endocrinology and Metabolism, Department of Internal Medicine, Biomedical Research Institute, \\ Pusan National University Hospital, Busan; ${ }^{4}$ Department of Internal Medicine, Chonnam National University Medical School, \\ Gwangju; ${ }^{5}$ Department of Internal Medicine, Chungnam National University Hospital, Chungnam National University College \\ of Medicine, Daejeon, Korea
}

Background: Hürthle cell carcinoma (HCC), a type of thyroid carcinoma, is rare in South Korea, and few studies have investigated its prognosis.

Methods: This long-term multicenter retrospective cohort study evaluated the clinicopathological features and clinical outcomes in patients with HCC who underwent thyroid surgery between 1996 and 2009.

Results: The mean age of the 97 patients included in the study was 50.3 years, and $26.8 \%$ were male. The mean size of the primary tumor was $3.2 \pm 1.8 \mathrm{~cm}$, and three (3.1\%) patients had distant metastasis at initial diagnosis. Ultrasonographic findings were available for 73 patients; the number of nodules with low-, intermediate-, and high suspicion was 28 (38.4\%), 27 (37.0\%), and 18 (24.7\%), respectively, based on the Korean-Thyroid Imaging Reporting and Data System. Preoperatively, follicular neoplasm (FN) or suspicion for $\mathrm{FN}$ accounted for $65.2 \%$ of the cases according to the Bethesda category, and $13 \%$ had malignancy or suspicious for malignancy. During a median follow-up of 8.5 years, eight (8.2\%) patients had persistent/recurrent disease, and none died of HCC. Older age, gross extrathyroidal extension (ETE), and widely invasive types of tumors were significantly associated with distant metastasis (all $P<0.01$ ). Gross ETE (hazard ratio [HR], 27.7; 95\% confidence interval [CI], 2.2 to 346.4; $P=0.01$ ) and widely invasive classification (HR, 6.5; 95\% CI, 1.1 to 39.4; $P=0.04$ ) were independent risk factors for poor disease-free survival (DFS).

Received: 14 June 2021, Revised: 26 July 2021, Accepted: 26 August 2021

Corresponding authors: Won Gu Kim

Division of Endocrinology and Metabolism, Department of Internal Medicine, Asan Medical Center, University of Ulsan College of Medicine, 88 Olympic-ro 43-gil, Songpa-gu, Seoul 05505, Korea

Tel: +82-2-3010-5883, Fax: +82-2-3010-6962, E-mail: wongukim@amc.seoul.kr

Mijin Kim

Division of Endocrinology and Metabolism, Department of Internal Medicine, Biomedical Research Institute, Pusan National University Hospital, 179 Gudeokro, Seo-gu, Busan 49241, Korea

Tel: +82-51-240-7738, Fax: +82-51-254-3237, E-mail: mijinkim08@gmail.com
Copyright $\odot 2021$ Korean Endocrine Society

This is an Open Access article distributed under the terms of the Creative Commons Attribution Non-Commercial License (https://creativecommons.org/ licenses/by-nc/4.0/) which permits unrestricted non-commercial use, distribution, and reproduction in any medium, provided the original work is properly cited.

*These authors contributed equally to this work. 
Conclusion: The long-term prognosis of HCC is relatively favorable in South Korea from this study, although this is not a nationwide data, and gross ETE and widely invasive cancer are significant prognostic factors for DFS. The diagnosis of HCC by ultrasonography and cytopathology remains challenging.

Keywords: Thyroid cancer; Hurthle cell carcinoma; Ultrasonography; Prognosis; Recurrence

\section{INTRODUCTION}

Hürthle cell carcinoma $(\mathrm{HCC})$ is a rare thyroid carcinoma that accounts for $3 \%$ to $4 \%$ of all malignant thyroid tumors [1,2]. Hürthle or oxyphilic cells are usually larger than the normal follicular cells and are characterized by abundant eosinophilic cytoplasm [3]. Thyroid nodules comprising $75 \%$ or more Hürthle cells are classified as Hürthle cell neoplasms (HCNs) [4]. Similar to follicular neoplasms (FNs), thyroid tumors demonstrating capsular and/or vascular invasion, lymph node (LN) metastasis, or presence of distant metastasis are diagnosed as HCC [4,5]. Previously, HCC was classified as an oxyphilic variant of follicular thyroid cancers, by the World Health Organization (WHO) [6]. However, due to their distinct genetic expression, unique pathologic characteristics, and clinical behavior, HCCs have been reclassified as a separate subtype of differentiated thyroid cancer (DTC) $[7,8]$.

In general, $\mathrm{HCC}$ has demonstrated more aggressive clinical behavior than other DTCs, in terms of more extrathyroidal extension (ETE), distant metastasis, less iodine-avid, and higher rates of mortality [9-11]. A population-level analysis using the Surveillance, Epidemiology, and End Results database reported that patients with HCC were older, presented with larger tumors, and had higher disease-specific mortality than patients with DTC [9]. However, not all studies have demonstrated consistent results [12-14]. As the prevalence of HCC is lower in regions with high iodine intakes, such as South Korea and Japan, limited studies have addressed the prognosis of patients with HCC in South Korea [15,16]. Considering the relatively small number of patients and a short follow-up period in these studies, a study that can fill this gap is necessary.

In addition, preoperative diagnosis of HCC by ultrasonography (US) and fine-needle aspiration (FNA) cytology is challenging. Until now, several studies have reported the imaging and cytopathology findings of HCC [16-18]. However, preoperative US and pathological features have not been elucidated because the number of patients with HCC included in these studies were small, and Hürthle cell adenoma was also included in the analysis.
Therefore, we aimed to evaluate the clinicopathological characteristics and long-term clinical outcomes in patients with HCC in a multi-institutional cohort in South Korea. Furthermore, we evaluated the prognostic factors that might be associated with distant metastasis at the time of diagnosis and persistent/recurrent disease during follow-up in patients with HCC.

\section{METHODS}

\section{Patients}

From January 1996 to December 2013, a total of 97 patients with HCC underwent thyroid surgery at five tertiary care hospitals in South Korea. All patients included in this retrospective multicenter study were confirmed to have HCC by histopathology and were aged $>18$ years. The study protocol was approved by the relevant Institutional Review Boards (Asan Medical Center, 2021-0568; Ulsan University Hospital, 2016-12-031; Pusan National University Hospital, 2105-038-013; Chonnam National University Hwasun Hospital, CNUHH-2017-053; and Chungnam National University Hospital, CNUH 2017-01-018). The need for informed consent was waived due to the retrospective design of the study.

\section{Neck US examination and evaluation of the finding}

The findings on the preoperative US of the neck were retrospectively reviewed by an experienced endocrinologist and radiologist at each center. US findings of the target nodule were evaluated according to the Korean Thyroid Imaging Reporting and Data System (K-TIRADS) [19]. The internal content of the nodule was categorized according to the ratio of the cystic to the solid portion within a nodule (e.g., predominantly solid, $>10 \%$ and $\leq 50 \%$ cystic). Echogenicity of the nodule was defined as a hypoechogenic pattern compared with an echogenicity pattern in the thyroid parenchyma and a marked hypoechogenic pattern as compared with that of the strap muscle. The nodule orientation was categorized as parallel or non-parallel. The tumor margins were classified as well-defined smooth, spiculated, or illdefined. Calcifications were categorized as microcalcification (calcification foci $\leq 1 \mathrm{~mm}$ ), macrocalcification (calcification 
foci $>1 \mathrm{~mm}$ ), or none. Preoperative cytopathology of the nodule was reported according to the 2017 Bethesda system [20].

\section{Follow-up protocol and definitions}

After thyroid surgery, subsequent radioactive iodine (RAI) therapy was administered according to guideline and physician's decision $[21,22]$. All patients were regularly followed up every 6 to 12 months. Physical examinations such as palpation of the neck and blood investigations, including thyroid function test, serum thyroglobulin, and anti-thyroglobulin antibody levels, were performed at each visit. Neck US was performed every 12 to 24 months, and US-guided FNA cytology was performed when there were abnormal findings on the US. Additional diagnostic imaging studies including neck/chest computed tomography or $18 \mathrm{~F}$-fluorodeoxyglucose-positron emission tomography were performed as needed such as increasing serum thyroglobulin [23].

Persistence/recurrence of disease was defined as the appearance of pathologically proven malignant tissue and/or appearance of metastatic lesions. Synchronous distant metastasis was defined as with persistent disease during follow-up. Diseasefree survival (DFS) was defined as the time from the date of surgery until last follow-up or recurrence. Disease-specific mortality was defined as death from HCC, and overall-mortality was defined as death from all causes.

\section{Statistical analyses}

Statistical analyses were performed using the R program version 3.5.1 (R Foundation for Statistical Computing, Vienna, Austria; http:/www.R-project.org), and figures were constructed using Graph Pad Prism version 5.0 (GraphPad Software, SanDiego, CA, USA; http:/www.graphpad.com). Continuous variables are presented as mean \pm standard deviation or medians and interquartile ranges (IQRs), and categorical variables are presented as numbers (percentages). We used the $t$ test and Kruskal-Wallis rank sum test to compare continuous variables and chi-square test to compare categorical variables. The Cox proportional hazard model was used to analyze the prognostic factors associated with DFS, presented as hazard ratios (HRs), 95\% confidence intervals (CIs), and $P$ values. Statistical significance was set at $P<0.05$.

\section{RESULTS}

\section{Baseline characteristics and clinical outcome of patients with HCC}

Table 1 presents the baseline characteristics of the 97 patients

\begin{tabular}{|c|c|}
\hline Characteristic & Value \\
\hline Number of patients & 97 \\
\hline Age, yr & $50.3 \pm 13.4$ \\
\hline Male sex & $26(26.8)$ \\
\hline Primary tumor size, $\mathrm{cm}$ & $3.2 \pm 1.8$ \\
\hline$\leq 2$ & $34(35.0)$ \\
\hline$>2$ and $\leq 4$ & $38(39.2)$ \\
\hline$>4$ & $25(25.8)$ \\
\hline \multicolumn{2}{|l|}{ Extra-thyroidal extension } \\
\hline Microscopic & $12(12.4)$ \\
\hline Gross & $3(3.1)$ \\
\hline \multicolumn{2}{|l|}{ Vascular invasion } \\
\hline Limited vascular invasion ( $<4$ foci) & $21(21.6)$ \\
\hline Extensive vascular invasion ( $\geq 4$ foci) & $3(3.1)$ \\
\hline \multicolumn{2}{|l|}{ WHO classification } \\
\hline Minimally invasive & $73(75.3)$ \\
\hline Encapsulated angio-invasive & $19(19.6)$ \\
\hline Widely invasive & $5(5.1)$ \\
\hline Cervical LN metastasis & $2(2.1)$ \\
\hline \multicolumn{2}{|l|}{ TNM stage (7th) } \\
\hline Stage I & $50(51.5)$ \\
\hline Stage II & $20(20.6)$ \\
\hline Stage III & $24(24.7)$ \\
\hline Stage IV & $3(3.1)$ \\
\hline \multicolumn{2}{|l|}{ TNM stage (8th) } \\
\hline Stage I & $82(84.5)$ \\
\hline Stage II & $12(12.4)$ \\
\hline Stage III & 0 \\
\hline Stage IV & $3(3.1)$ \\
\hline Distant metastasis & $3(3.1)$ \\
\hline Total thyroidectomy & $48(49.5)$ \\
\hline Radioiodine ablation & $40(41.2)$ \\
\hline
\end{tabular}

with HCC. The mean age of the patients at the time of initial surgery was $50.3 \pm 13.4$ years, and $26.8 \%$ were males. The mean size of the primary tumor was $3.2 \pm 1.8 \mathrm{~cm}$, and $25.8 \%$ of the tumors were larger than $4 \mathrm{~cm}$. Microscopic ETE and gross ETE were found in $12(12.4 \%)$ and three $(3.1 \%)$ patients, respectively. Vascular invasion was found in 24 (24.7\%) patients, among whom three had extensive vascular invasion ( $\geq 4$ foci). 
Based on the WHO classification, the number of patients with minimally invasive, encapsulated angio-invasive, and widely invasive tumors was 73 (75.3\%), 19 (19.6\%), and five (5.1\%), respectively. At the time of diagnosis, two (2.1\%) patients had cervical LN metastasis, and three (3.1\%) patients had distant metastasis. Based on the 8th edition of the American Joint Committee on Cancer/Union for International Cancer Control tumornode-metastasis (TNM) staging system, the number of patients with stage I, II, III, and IV classification was 82 (84.5\%), 12 (12.4\%), 0, and three (3.1\%), respectively. Forty-eight (49.5\%) patients underwent total thyroidectomy, and 40 of them received subsequent RAI therapy. During a median follow-up of 8.5 years (IQR, 5.2 to 12.8), eight (8.2\%) patients had persistent/recurrent disease. Among eight patients, four patients had local recurrence such as operation bed and cervical LN metastasis. One patient had newly detected subcutaneous metastasis and the other three had persistent metastasis in lung and bone. Nine (9.3\%) patients died from other causes, and none of them died of HCC.

\section{Ultrasonographic and cytopathologic features of HCC}

Of the 97 patients, US findings of HCC were available for 73 patients (Table 2). Most ( $n=62,84.9 \%)$ were solid nodules, and in terms of echogenicity, 40 (54.8\%) were hypoechoic, and nine $(12.3 \%)$ were markedly hypoechoic. Ten nodules $(13.7 \%)$ had non-parallel orientation, and 68 (93.2\%) had smooth margins. In terms of calcification, seven (9.6\%) had microcalcification, and nine $(12.3 \%)$ had macrocalcification. Based on the K-TIRADS, 28 (38.4\%), 27 (37.0\%), and 18 (24.7\%) patients were classified into the low-, intermediate-, and high suspicion categories, respectively (Fig. 1A).

Among all patients included in the study, results of preoperative FNA or core needle biopsy (CNB) were available for 92 patients. According to the Bethesda category, FN or suspected FN was the most common and was observed in $60(65.2 \%)$ patients (Table 2, Fig. 1B). Atypia of undetermined significance/ follicular lesion of undetermined significance, suspicious for malignancy, and malignancy accounted for $8.7 \%, 5.4 \%$, and $7.6 \%$ of the cases, respectively. The FNA/CNB results according to the K-TIRADS category are shown in Fig. 1C. The proportion of tumors with Bethesda category IV-VI increased toward from low to high suspicion nodule, accounts for $74.1 \%$, $80.8 \%$, and $83.4 \%$, respectively. CNB was performed in 11 out of 92 patients and there was no significant difference between pathology according to FNA or CNB $(P=0.24)$.

\begin{tabular}{lc} 
Table 2. Ultrasonographic Features of Hürthle Cell Carcinoma \\
\hline Characteristic & Value \\
\hline Number of patients & 73 \\
Composition & $62(84.9)$ \\
Solid & $11(15.1)$ \\
Predominantly solid & \\
Echogenicity & $9(12.3)$ \\
Markedly hypoechoic & $40(54.8)$ \\
Hypoechoic & $24(32.9)$ \\
Isoechoic & $10(13.7)$ \\
Non-parallel & \\
Margin & $68(93.2)$ \\
Smooth & $3(4.1)$ \\
Spiculated & $2(2.7)$ \\
Ill-defined & \\
Calcification & $18(24.7)$ \\
Microcalcification & \\
Macrocalcification & $7(9.6)$ \\
K-TIRADS & \\
Low suspicion & $9(65.2)$ \\
Intermediate suspicion & $7(7.4)$ \\
FNigh suspicion & $28(38.4)$ \\
Nondiagnostic & $27(37.0)$ \\
Benign & \\
AUS/FL or suspicious for FN & \\
\hline & \\
Malignancy & \\
\hline
\end{tabular}

Values are expressed as number (\%).

K-TIRADS, Korean-Thyroid Imaging Reporting and Data System; FNA, fine needle aspiration; CNB, core needle biopsy; AUS, atypia of undetermined significant; FLUS, follicular lesion of undetermined significance; FN, follicular neoplasm.

${ }^{a} \mathrm{FNA} / \mathrm{CNB}$ result was available in 92 patients.

\section{Clinicopathological features associated with synchronous distant metastasis}

We compared the clinicopathological features between patients with and without distant metastasis at initial surgery (Supplemental Table S1). Patients with distant metastasis were significantly older than those without distant metastasis (68.5 years vs. 49.8 years, $P<0.01)$. There was no significant difference in the sex ratio and primary tumor size between the two groups. Two $(66.7 \%)$ patients in the distant metastasis group had gross ETE, 

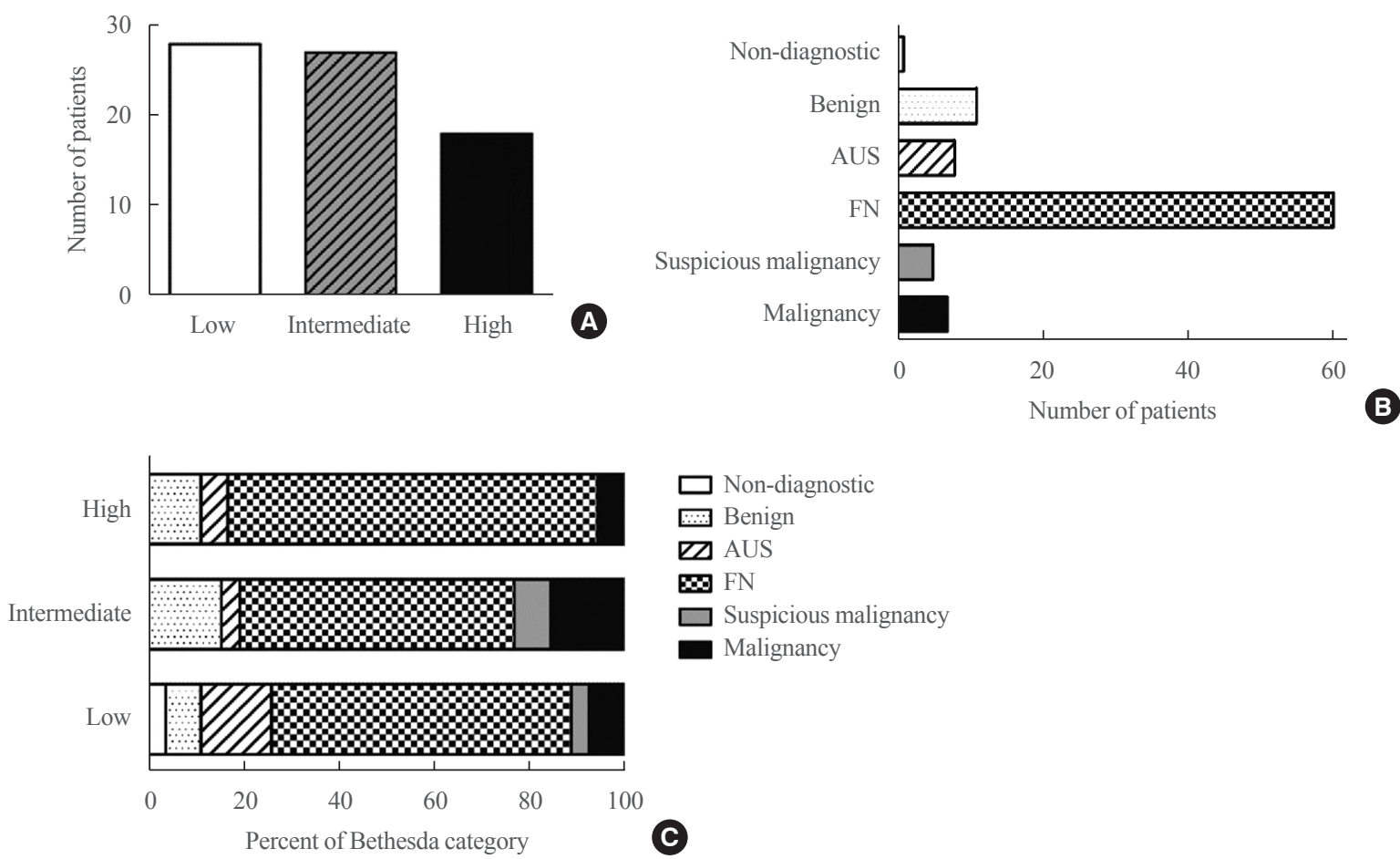

Fig. 1. (A) Ultrasonographic findings based on the Korean-Thyroid Imaging Reporting and Data System in patients with Hürthle cell carcinoma. (B) Preoperative fine needle aspiration or core needle biopsy based on Bethesda category in patients with Hürthle cell carcinoma. (C) Proportion of Bethesda category according to ultrasonographic feature. AUS, atypia of undetermined significant; FN, follicular neoplasm.

Table 3. Clinicopathological Features Associated with Disease-Free Survival in Patients with Hürthle Cell Carcinoma

\begin{tabular}{|c|c|c|c|c|}
\hline \multirow{2}{*}{ Variable } & \multicolumn{2}{|c|}{ Univariate } & \multicolumn{2}{|c|}{ Multivariate } \\
\hline & $\mathrm{HR}(95 \% \mathrm{CI})$ & $P$ value & $\operatorname{HR}(95 \% \mathrm{CI})$ & $P$ value \\
\hline Older age $(\geq 55 \mathrm{yr})$ & $6.2(1.2-30.9)$ & 0.03 & $2.8(0.4-17.8)$ & 0.28 \\
\hline Male sex & $1.9(0.5-8.1)$ & 0.37 & - & - \\
\hline Larger size $(>4 \mathrm{~cm})$ & $0.8(0.2-4.1)$ & 0.81 & - & - \\
\hline Gross ETE (ref. no ETE and microscopic ETE) & $82.5(7.4-923.5)$ & $<0.01$ & $27.7(2.2-346.4)$ & 0.01 \\
\hline \multicolumn{5}{|l|}{ WHO classification (ref. minimally invasive) } \\
\hline Encapsulated angio-invasive & $2.4(0.4-14.8)$ & 0.33 & & \\
\hline Widely invasive & $17.3(3.5-85.9)$ & $<0.01$ & $6.5(1.1-39.4)$ & 0.04 \\
\hline Cervical LN metastasis & $1.1 \mathrm{e}-07(-\mathrm{INF})$ & 0.99 & - & - \\
\hline Total thyroidectomy & $3.1(0.6-15.3)$ & 0.17 & - & - \\
\hline Radioiodine ablation therapy & $1.4(0.3-5.6)$ & 0.64 & - & - \\
\hline
\end{tabular}

which was significantly higher than that $(1.1 \%)$ in patients without distant metastasis $(P<0.01)$. According to the WHO classification, widely invasive (66.7\%) and encapsulated angio-invasive $(33.3 \%)$ tumors were observed more frequently in patients with distant metastasis than in those without distant metastasis $(P<0.01)$.
We also evaluated the clinical and ultrasonographic features of patients who had features of high risk of recurrent disease such as gross ETE, LN metastasis, distant metastasis, and widely invasive tumor. Because some factors overlap, there are 9 patients with high-risk of recurrent disease (Supplemental Table S2). They were older $(67.5$ years vs. 49.8 years, $P<0.01)$ than 
those with low risk of recurrence and showed a higher rate of total thyroidectomy ( $88.9 \%$ vs. $45.5 \%, P=0.03)$.

\section{Clinicopathological features associated with DFS}

To identify the clinicopathological factors associated with DFS in patients with $\mathrm{HCC}$, univariate and multivariate analyses were performed (Table 3 ). Older age ( $\geq 55$ years, HR, 6.2; 95\% CI, 1.2 to $30.9 ; P=0.03$ ), gross ETE (HR, 82.5; 95\% CI, 7.4 to 923.5; $P<0.01$ ), and widely invasive tumors according to the WHO classification (HR, 17.3; 95\% CI, 3.5 to $85.9 ; P<0.01)$ were significantly associated with poorer DFS. In multivariate analysis, gross ETE (HR, 27.7; 95\% CI, 2.2 to 346.4; $P=0.01$ ) and widely invasive tumors (HR, 6.5; 95\% CI, 1.1 to 39.4 ; $P=0.04)$ were independent risk factors associated with DFS.

\section{DISCUSSION}

$\mathrm{HCC}$ is a relatively uncommon thyroid malignancy in iodinesufficient regions. According to a single-center study in South Korea, among patients diagnosed with thyroid cancer during 1995 to 2005 , HCC accounted for only 1\% [15]. In this multicenter cohort study in South Korea, where HCC is relatively rare, we evaluated the clinical outcome of patients with HCC. Persistent/recurrent disease was observed in $8.2 \%$ of the patients, and none died of HCC during a median follow-up of 8.5 years. Distant metastasis was significantly associated with older age, gross ETE, and widely invasive cancer. We found that the clinicopathological factors associated with DFS, gross ETE, and widely invasive cancer were independent risk factors associated with poor DFS.

Previous studies reported that HCC is associated with a more aggressive clinical behavior, for example, higher rates of distant metastasis, a higher proportion of iodine resistant tumors, and higher mortality than other DTCs [9-11,24]. However, this has been challenged in several studies, which reported that HCC had a similar outcome as follicular thyroid carcinoma [12$14,16]$. In the latest population-level analysis that included 3,111 patients with $\mathrm{HCC}$, the overall survival rate was $82.1 \%$, and the disease-specific survival rate was 94.1\% [9]. A similar survival outcome was seen in a recently reported single-center data of 239 patients. The 5-year overall survival rates and disease-specific survival rates were $89.4 \%$ and $94.6 \%$, respectively [25]. In our study, the overall survival rate was $91.7 \%$, and none of the patients died of thyroid cancer during a median follow-up of 8.5 years. Furthermore, the proportion of patients with persistent/ recurrent disease was $8.2 \%$, which was lower than reported in another study (12.1\%) with a similar follow-up period [25]. The favorable prognosis observed in our study compared to that in the study by Oluic et al. [25] study, might be due to a low proportion of widely invasive tumors (5\% vs. 33\%) and larger size tumors ( $>4 \mathrm{~cm}, 26 \%$ vs. $54 \%$ ). Furthermore, these results may be related to the early diagnosis and treatment of cancer in this study. Given the rarity of this condition, more studies with larger sample sizes are necessary to validate the findings.

Distant metastasis was found in $4.7 \%$ to $11.0 \%$ of the patients with HCC in large studies conducted in the United States in recent times $[9,11,26]$. Previously, Kim et al. [15] reported that distant metastasis was found in $2.5 \%$ of the patients with $\mathrm{HCC}$ in a study that included some patients from the current study. Similarly, the frequency of synchronous distant metastasis was $3.1 \%$ in this study. The reason for the relatively low incidence of distant metastasis in South Korea is unclear. We found that distant metastasis was more frequent in patients with higher age, gross ETE, and widely invasive cancer, which is consistent with previous reports [16].

In this study, we found that higher age, gross ETE, and widely invasive cancer were associated with poor DFS, although only gross ETE and widely invasive cancer were independent risk factors in multivariate analysis. The prognosis of HCC differs between minimally and widely invasive types. Chindris et al. [26] reported that neither recurrence nor disease-specific mortality occurred in 39 patients with minimally invasive HCC, while $62.7 \%$ of patients with widely invasive disease had a clinical recurrence or death. Furthermore, widely invasive type, male sex, older age, and higher TNM stage were also thought to be prognostic factors in patients with $\mathrm{HCC}$; however, these studies mainly focused on survival $[9,11,26]$. Age was a wellknown prognostic factor associated with survival, but not with recurrence.

One of the strengths of this study is that we evaluated the US features of HCC, which are not well known. Due to the rarity of HCC, the US findings have been reported only in a few studies or were analyzed along with those of Hürthle cell adenoma [18]. In the current study, we found that HCC usually showed a solid hypoechoic appearance on US, and on the preoperative cytopathology, FN or suspicious FN was most commonly observed, accounting for $65.2 \%$ of the cases according to the Bethesda category. The diagnosis of HCC is challenging because the results of the FNA of HCC are usually non-conclusive for malignancy. The risk of malignancy is only $15 \%$ to $30 \%$ when $\mathrm{HCN}$ is conclusively detected on cytopathology [21,27-29]. In a previous cohort study in South Korea, only $67 \%$ of the patients di- 
agnosed with HCC after surgery showed malignancy on preoperative FNA. Therefore, diagnostic thyroid surgery is still necessary based on the tumor characteristics, including US features $[16,17,30,31]$. This study further strengthens this concept because only $24.7 \%$ of HCCs were classified as high-suspicion nodules on the preoperative US, and $21.8 \%$ did not show malignancy on preoperative cytopathology in patients who were confirmed to have HCC after surgery.

There were some limitations in this study. First, it was a retrospective study; hence, a potential selection bias might exist. Second, we could not evaluate the clinical factors associated with disease-specific mortality since no patient died of HCC. Third, there might be some differences in the follow-up strategies between the five different centers. However, this is the first multicenter cohort study to evaluate the prognosis of $\mathrm{HCC}$ in South Korea.

In summary, to the best of our knowledge, the current study is the largest analysis of patients with HCC in South Korea. The diagnosis of HCC based on US and cytopathology is still challenging. The long-term prognosis of HCC is relatively favorable, with the persistence/recurrence rate of $8.2 \%$ during a median follow-up of 8.5 years. Gross ETE and widely invasive cancer were independent risk factors associated with poor DFS.

\section{CONFLICTS OF INTEREST}

No potential conflict of interest relevant to this article was reported.

\section{ACKNOWLEDGMENTS}

This study was supported by a Korean Endocrine Society of EnM Research Award 2020.

\section{AUTHOR CONTRIBUTIONS}

Conception or design: W.G.K. Acquisition, analysis, or interpretation of data: M.J., E.S.K., B.H.K., H.K.K., Y.E.K., M.J.J., T.Y.K., H.C.K., W.B.K., Y.K.S. Drafting the work or revising: M.J., E.S.K. Final approval of the manuscript: M.K.

\section{ORCID}

Meihua Jin https://orcid.org/0000-0003-0196-631X

Eun Sook Kim https://orcid.org/0000-0002-9311-4642

Mijin Kim https://orcid.org/0000-0002-1538-8859
Won Gu Kim https://orcid.org/0000-0002-8404-7759

\section{REFERENCES}

1. Hundahl SA, Fleming ID, Fremgen AM, Menck HR. A National Cancer Data Base report on 53,856 cases of thyroid carcinoma treated in the U.S., 1985-1995 [see commetns]. Cancer 1998;83:2638-48.

2. Hundahl SA, Cady B, Cunningham MP, Mazzaferri E, McKee RF, Rosai J, et al. Initial results from a prospective cohort study of 5583 cases of thyroid carcinoma treated in the United States during 1996. U.S. and German Thyroid Cancer Study Group: an American College of Surgeons Commission on Cancer Patient Care Evaluation study. Cancer 2000;89:202-17.

3. Mete O, Asa SL. Oncocytes, oxyphils, Hurthle, and Askanazy cells: morphological and molecular features of oncocytic thyroid nodules. Endocr Pathol 2010;21:16-24.

4. Montone KT, Baloch ZW, LiVolsi VA. The thyroid Hurthle (oncocytic) cell and its associated pathologic conditions: a surgical pathology and cytopathology review. Arch Pathol Lab Med 2008;132:1241-50.

5. Kure S, Ohashi R. Thyroid Hurthle cell carcinoma: clinical, pathological, and molecular features. Cancers (Basel) 2020; $13: 26$.

6. Hedinger C, Williams ED, Sobin LH. The WHO histological classification of thyroid tumors: a commentary on the second edition. Cancer 1989;63:908-11.

7. Ganly I, Ricarte Filho J, Eng S, Ghossein R, Morris LG, Liang Y, et al. Genomic dissection of Hurthle cell carcinoma reveals a unique class of thyroid malignancy. J Clin Endocrinol Metab 2013;98:E962-72.

8. LIoyd RV, Osamura RY, Kloppel G, Rosai J. WHO classification of tumours of endocrine organs. 10th ed. Lyon: International Agency for Research on Cancer; 2017.

9. Goffredo P, Roman SA, Sosa JA. Hurthle cell carcinoma: a population-level analysis of 3311 patients. Cancer 2013;119: 504-11.

10. Phitayakorn R, McHenry CR. Follicular and Hurthle cell carcinoma of the thyroid gland. Surg Oncol Clin N Am 2006;15:603-23.

11. Lopez-Penabad L, Chiu AC, Hoff AO, Schultz P, Gaztambide $\mathrm{S}$, Ordonez NG, et al. Prognostic factors in patients with Hurthle cell neoplasms of the thyroid. Cancer 2003;97: 1186-94.

12. Nagar S, Aschebrook-Kilfoy B, Kaplan EL, Angelos P, GroCopyright (C) 2021 Korean Endocrine Society 
gan RH. Hurthle cell carcinoma: an update on survival over the last 35 years. Surgery 2013;154:1263-71.

13. Khafif A, Khafif RA, Attie JN. Hurthle cell carcinoma: a malignancy of low-grade potential. Head Neck 1999;21: 506-11.

14. Bhattacharyya N. Survival and prognosis in Hurthle cell carcinoma of the thyroid gland. Arch Otolaryngol Head Neck Surg 2003;129:207-10.

15. Kim WG, Yim JH, Kim EY, Kim TY, Gong G, Yoon JH, et al. Preoperative cytological diagnosis of follicular thyroid carcinoma and Huthle cell carcinoma. J Korean Thyroid Assoc 2010;3:149-54.

16. Kim WG, Kim TY, Kim TH, Jang HW, Jo YS, Park YJ, et al. Follicular and Hurthle cell carcinoma of the thyroid in iodine-sufficient area: retrospective analysis of Korean multicenter data. Korean J Intern Med 2014;29:325-33.

17. Kim TH, Lim JA, Ahn HY, Lee EK, Min HS, Kim KW, et al. Tumor size and age predict the risk of malignancy in Hurthle cell neoplasm of the thyroid and can therefore guide the extent of initial thyroid surgery. Thyroid 2010;20:122934.

18. Santana NO, Freitas RM, Marcos VN, Chammas MC, Camargo RY, Schmerling CK, et al. Diagnostic performance of thyroid ultrasound in Hurthle cell carcinomas. Arch Endocrinol Metab 2019;63:300-5.

19. Shin JH, Baek JH, Chung J, Ha EJ, Kim JH, Lee YH, et al. Ultrasonography diagnosis and imaging-based management of thyroid nodules: revised Korean Society of Thyroid Radiology consensus statement and recommendations. Korean J Radiol 2016;17:370-95.

20. Cibas ES, Ali SZ. The 2017 Bethesda system for reporting thyroid cytopathology. Thyroid 2017;27:1341-6.

21. Haugen BR, Alexander EK, Bible KC, Doherty GM, Mandel SJ, Nikiforov YE, et al. 2015 American Thyroid Association management guidelines for adult patients with thyroid nodules and differentiated thyroid cancer: the American Thyroid Association Guidelines Task Force on thyroid nodules and differentiated thyroid cancer. Thyroid 2016;26:1133.

22. American Thyroid Association (ATA) Guidelines Taskforce on Thyroid Nodules and Differentiated Thyroid Cancer,
Cooper DS, Doherty GM, Haugen BR, Kloos RT, Lee SL, et al. Revised American Thyroid Association management guidelines for patients with thyroid nodules and differentiated thyroid cancer. Thyroid 2009;19:1167-214.

23. Jin M, Kim ES, Kim BH, Kim HK, Yi HS, Jeon MJ, et al. Clinical implication of World Health Organization classification in patients with follicular thyroid carcinoma in South Korea: a multicenter cohort study. Endocrinol Metab (Seoul) 2020;35:618-27.

24. Mills SC, Haq M, Smellie WJ, Harmer C. Hurthle cell carcinoma of the thyroid: retrospective review of 62 patients treated at the Royal Marsden Hospital between 1946 and 2003. Eur J Surg Oncol 2009;35:230-4.

25. Oluic B, Paunovic I, Loncar Z, Djukic V, Diklic A, Jovanovic M, et al. Survival and prognostic factors for survival, cancer specific survival and disease free interval in 239 patients with Hurthle cell carcinoma: a single center experience. BMC Cancer 2017;17:371.

26. Chindris AM, Casler JD, Bernet VJ, Rivera M, Thomas C, Kachergus JM, et al. Clinical and molecular features of Hurthle cell carcinoma of the thyroid. J Clin Endocrinol Metab 2015;100:55-62.

27. Baloch ZW, LiVolsi VA, Asa SL, Rosai J, Merino MJ, Randolph G, et al. Diagnostic terminology and morphologic criteria for cytologic diagnosis of thyroid lesions: a synopsis of the National Cancer Institute Thyroid Fine-Needle Aspiration State of the Science Conference. Diagn Cytopathol 2008;36:425-37.

28. Sorrenti S, Trimboli P, Catania A, Ulisse S, De Antoni E, D'Armiento M. Comparison of malignancy rate in thyroid nodules with cytology of indeterminate follicular or indeterminate Hurthle cell neoplasm. Thyroid 2009;19:355-60.

29. Pu RT, Yang J, Wasserman PG, Bhuiya T, Griffith KA, Michael CW. Does Hurthle cell lesion/neoplasm predict malignancy more than follicular lesion/neoplasm on thyroid fineneedle aspiration? Diagn Cytopathol 2006;34:330-4.

30. Thompson NW, Dunn EL, Batsakis JG, Nishiyama RH. Hurthle cell lesions of the thyroid gland. Surg Gynecol Obstet 1974;139:555-60.

31. Yutan E, Clark OH. Hurthle cell carcinoma. Curr Treat Options Oncol 2001;2:331-5. 